\title{
OPTIMUM PROBLEMS WITH MEASURABLE SET-VALUED CONSTRAINTS*
}

\author{
ZSOLT PÁLES ${ }^{\dagger}$ AND VERA ZEIDAN ${ }^{\ddagger}$
}

\begin{abstract}
In this paper, we provide a complete analysis of second-order admissible variations to inequality-type constraints, which are given in terms of measurable set-valued functions whose images are closed convex sets with nonempty interior. As an application, we consider optimization problems where such constraints are present, and we deduce second-order necessary conditions for optimality.
\end{abstract}

Key words. measurable set-valued maps, convex sets in $L^{\infty}\left(\Omega, \mathbb{R}^{m}\right)$, support functional, tangent and normal cones, second-order admissible variations, second-order optimality conditions

AMS subject classifications. Primary, 90C48, 90C34; Secondary, 58C06, 47H04

PII. S1052623499350943

1. Introduction. Consider the following optimization problem:

$$
\text { Minimize } F_{0}(z) \text { subject to } E(z)=0, F(z) \leq 0, G(z) \in \mathbf{Q} \text {, }
$$

where $F_{0}: \mathcal{D} \rightarrow \mathbb{R}, E: \mathcal{D} \rightarrow Y, F: \mathcal{D} \rightarrow \mathbb{R}^{p}, G: \mathcal{D} \rightarrow X$, and $X, Y, Z$ are Banach spaces, $\mathcal{D} \subset Z$ is nonempty and open, and $\mathbf{Q} \subset X$ is a closed convex set with nonempty interior.

The prototype of such problems arises, for instance, in optimal control theory with control constraints in the inclusion form $x(t) \in Q(t)$ (for all $t \in \Omega$ ), where $Q$ is a measurable set-valued map with closed convex nonempty interior images on the complete finite measure space $(\Omega, \mathcal{A}, \mu)$.

Better understanding of optimality conditions is an ongoing research program for several researchers. This question is of great value in theory and in applications. Usually, such conditions must be given in terms of the original data of the problem and, in the context of necessity, are expected to be as strong as they can be.

In 1988, Kawasaki [11], [12] discovered, for the problem $(\mathcal{P})$, where $\mathbf{Q}$ is a cone, second-order necessary conditions that contain an extra term manifesting the presence of infinitely many inequalities in the constraint $G(z) \in \mathbf{Q}$. This phenomenon is known as the "envelope-like effect." Such result was generalized by Cominetti in [4]. Both results assumed a Mangasarian-Fromovitz-type condition.

In [18] the authors have generalized the previous results in [11], [12], and [4] to the nondifferentiable case without assuming a Mangasarian-Fromowitz condition. The second-order admissible variation set used therein (defined first by Dubovitskii and Milyutin in [7], [8]) is described in the following definition.

Definition. Let $X$ be a normed space, $\mathbf{Q} \subset X, x \in \mathbf{Q}$, and $d \in X$. A vector $v \in X$ is called a second-order admissible variation of $\mathbf{Q}$ at $x$ in the direction $d$ if

*Received by the editors January 25, 1999; accepted for publication (in revised form) May 11, 2000; published electronically October 18, 2000.

http://www.siam.org/journals/siopt/11-2/35094.html

$\dagger$ Institute of Mathematics and Informatics, Debrecen University, H-4010 Debrecen, Pf. 12, Hungary (pales@math.klte.hu). This author's research was supported by Hungarian National Foundation for Scientific Research (OTKA) grant T-030082 and by Hungarian Higher Education Research and Development Fund (FKFP) grant 0310/1997.

${ }^{\ddagger}$ Department of Mathematics, Michigan State University, East Lansing, MI 48824 (zeidan@ math.msu.edu). This author's research was supported partially by the Department of Mathematics at Michigan State University. 
there exists $\bar{\varepsilon}>0$ such that

$$
x+\varepsilon d+\varepsilon^{2}(v+w) \in \mathbf{Q} \quad \text { for all } 0<\varepsilon<\bar{\varepsilon},\|w\|<\bar{\varepsilon}, w \in X .
$$

The set of all such variations is denoted by $V(x, d \mid \mathbf{Q})$. It follows directly from the definition that $V(x, d \mid \mathbf{Q})$ is an open set. If $\mathbf{Q}$ is also convex, then $V(x, d \mid \mathbf{Q})$ is convex as well. Results related to those in [18] were obtained by Maruyama [14, Theorem $3.2]$, where Neustadt's derivative was used to handle the nonsmoothness of data.

In order to derive meaningful second-order optimality conditions, it is imperative to choose directions $d$ that guarantee the nonemptiness of $V(x, d \mid \mathbf{Q})$. Such directions $d \in X$ are labeled as the critical directions of $\mathbf{Q}$ at $x$ and form a set called critical direction cone to $\mathbf{Q}$ at $x$. Throughout this paper, this cone will be denoted by $C(x \mid \mathbf{Q})$. It can be easily seen that $C(x \mid \mathbf{Q})$ is a convex set such that

$$
\text { cone }(Q-x) \subset C(x \mid Q) \subset \overline{\operatorname{cone}}(Q-x) .
$$

In order to recall the first- and second-order necessary conditions for $(\mathcal{P})$, obtained in [18, Corollary 2], we need to introduce the following notation and notions.

- A point $\hat{z} \in \mathcal{D}$ is called an admissible point for $(\mathcal{P})$ if $E(\hat{z})=0, F(\hat{z}) \leq 0$, and $G(\hat{z}) \in \mathbf{Q}$ hold. A point $\hat{z} \in \mathcal{D}$ is a solution (local minimum) of the problem if it is admissible and there exists a neighborhood $U$ of $\hat{z}$ such that $F_{0}(z) \geq F_{0}(\hat{z})$ for all admissible points $z \in U$.

- A point $\hat{z} \in \mathcal{D}$ is called a regular point for $(\mathcal{P})$ if

$\left(R_{1}\right) F_{0}, F=\left(F_{1}, \ldots, F_{p}\right)$ are locally Lipschitz at $\hat{z}$;

$\left(R_{2}\right) G$ is strictly Fréchet differentiable at $\hat{z}$;

$\left(R_{3}\right) E$ is strictly Fréchet differentiable at $\hat{z}$ and the range of the linear operator $E^{\prime}(\hat{z})$ is a closed subspace of $Y$.

If $F_{i}(i=0, \ldots, p)$ is locally Lipschitz at $\hat{z}$, then the expression

$$
F_{i}^{o}(\hat{z} ; y):=\limsup _{(z, \varepsilon) \rightarrow(\hat{z}, 0+)} \frac{F_{i}(z+\varepsilon y)-F_{i}(z)}{\varepsilon}
$$

is finite and will be called Clarke's generalized directional derivative in the direction $y$. The corresponding generalized gradient $\partial F_{i}(\hat{z})$ is defined by

$$
\partial F_{i}(\hat{z}):=\left\{z^{*} \in Z^{*}:\left\langle z^{*}, z\right\rangle \leq F_{i}^{o}(\hat{z} ; z) \text { for all } z \in Z\right\} .
$$

For properties of these notions, see [2].

Let $\hat{z}$ be an admissible regular point for $(\mathcal{P})$ and $d \in Z$.

- A vector $y \in Z$ is called a critical direction at $\hat{z}$ for $(\mathcal{P})$ if

$\left(C_{1}\right) F_{i}^{o}(\hat{z} ; y) \leq 0$ for all $i=0, \ldots, p$;

$\left(C_{2}\right) G^{\prime}(\hat{z}) y \in C(G(\hat{z}) \mid \mathbf{Q})$

$\left(C_{3}\right) E^{\prime}(\hat{z}) y=0$.

- A vector $y \in Z$ is called a regular direction at $\hat{z}$ for $(\mathcal{P})$ if

$\left(R_{4}\right)$ for all $i=0, \ldots, p$,

$$
F_{i}^{o \prime}(\hat{z}, y):=\limsup _{\varepsilon \rightarrow 0+} 2 \frac{F_{i}(\hat{z}+\varepsilon y)-F_{i}(\hat{z})-\varepsilon F_{i}^{o}(\hat{z} ; y)}{\varepsilon^{2}}
$$

is finite;

$\left(R_{5}\right)$ the second-order directional derivative of $L:=(G, E)$ 


$$
L^{\prime \prime}(\hat{z}, y):=\lim _{\varepsilon \rightarrow 0+} 2 \frac{L(\hat{z}+\varepsilon y)-L(\hat{z})-\varepsilon L^{\prime}(\hat{z}) y}{\varepsilon^{2}}
$$

exists.

Clearly, the zero vector is always a regular critical direction at $\hat{z}$ for $(\mathcal{P})$.

Now we are ready to state the result of [18, Corollary 2].

THEOREM 1.1. Let $\hat{z}$ be a regular local solution of the above problem $(\mathcal{P})$. Then, for all regular critical directions $y$, there correspond Lagrange multipliers $\lambda_{i} \geq 0(i=$ $0, \ldots, p), x^{*} \in X^{*}$, and $y^{*} \in Y^{*}$ which depend on $y$, such that at least one of them is different from zero and the following relations hold:

$$
\begin{aligned}
& \lambda_{i} F_{i}(\hat{z})=0 \quad \text { for all } i=1, \ldots, p \quad \text { and } \quad x^{*} \in N(G(\hat{z}) \mid \mathbf{Q}), \\
& \sum_{i=0}^{p} \lambda_{i} F_{i}^{o}(\hat{z} ; z)+\left\langle x^{*}, G^{\prime}(\hat{z}) z\right\rangle+\left\langle y^{*}, E^{\prime}(\hat{z}) z\right\rangle \geq 0 \quad \text { for } z \in Z,
\end{aligned}
$$

and

$$
\sum_{i=0}^{p} \lambda_{i} F^{o \prime}(\hat{z}, y)+\left\langle x^{*}, G^{\prime \prime}(\hat{z}, y)\right\rangle+\left\langle y^{*}, E^{\prime \prime}(\hat{z}, y)\right\rangle \geq 2 \delta^{*}\left(x^{*} \mid V\left(G(\hat{z}), G^{\prime}(\hat{z}) y \mid \mathbf{Q}\right)\right) .
$$

(Here $\delta^{*}$ stands for the support function and $N(x \mid \mathbf{Q})$ denotes the adjoint cone of $T(x \mid \mathbf{Q})$, that is, the cone of outward normals to the set $\mathbf{Q}$ at the point $x$ [24].)

We note that, using the Hahn-Banach theorem, the first-order condition (1.2) can also be expressed as an equality: There exist linear functionals $z_{i}^{*} \in \partial F_{i}(\hat{z})$ $(i=0, \ldots, p)$ such that

$$
\sum_{i=0}^{p} \lambda_{i} z_{i}^{*}+x^{*} \circ G^{\prime}(\hat{z})+y^{*} \circ E^{\prime}(\hat{z})=0 .
$$

Throughout this paper the term to the right-hand side of inequality (1.3) will be referred to as the extra term in the second-order condition.

Results along the line of Theorem 1.1 were obtained by Ioffe [10] and Penot [23] for the differentiable case and in the presence of a certain qualification condition.

Two important questions naturally surface from Theorem 1.1:

(i) How can we check the nonemptiness of $V(x, d \mid \mathbf{Q})$, that is, how can the critical cone $C(x \mid \mathbf{Q})$ be characterized, since otherwise the second-order optimality conditions would be satisfied trivially?

(ii) How can we evaluate the support function of $V(x, d \mid \mathbf{Q})$ ?

In order that $d$ be in $C(x \mid \mathbf{Q})$, it is only necessary that $\mathbf{Q}$ have a nonempty interior and that $d$ belong to $\overline{c o n e}(\mathbf{Q}-x)=T(x \mid \mathbf{Q})$, which is the tangent cone to $\mathbf{Q}$ at $x$. If $d \in$ cone $(\mathbf{Q}-x)$, then $V(x, d \mid \mathbf{Q})$ is nonempty and $V(x, d \mid \mathbf{Q})=$ cone $($ cone $($ int $\mathbf{Q}-x)-d$ ) (cf. $[18$, Theorem 4]). In this case the right-hand side in the second-order condition (1.3) vanishes. However, examples are provided by Kawasaki [11] in order to show that the necessary conditions with extra term, that is, when $d \in \overline{\operatorname{cone}}(\mathbf{Q}-x)$, handle situations that cannot be handled with previous results where $d$ is taken from $\operatorname{cone}(\mathbf{Q}-x)$. Thus, one has to consider also directions $d \in T(x \mid \mathbf{Q}) \backslash \operatorname{cone}(\mathbf{Q}-x)$. In this case the description of $V(x, d \mid \mathbf{Q})$ and the characterization of its nonemptiness are far from being trivial. 
A significant setting is the case when $\mathbf{Q}$ is a subset of $C\left(T, \mathbb{R}^{r}\right)$ defined by

$$
\mathbf{Q}=\sigma_{C}(Q)=:\left\{x \in C\left(T, \mathbb{R}^{r}\right) \mid x(t) \in Q(t) \text { for all } t \in T\right\},
$$

where $Q$ is a lower semicontinuous set-valued map whose images are closed, convex sets with nonempty interior, and $T$ is a compact Hausdorff space. The importance of this type of constraints stems from control problems with state constraints. This problem has been studied and a satisfactory answer to questions (i) and (ii) above have been provided in a recent paper by the authors [22].

Another case of great interest is when $\mathbf{Q}$ is a subset of $L^{\infty}\left(\Omega, \mathbb{R}^{m}\right)$ defined by

$$
\mathbf{Q}=\sigma_{\infty}(Q):=\left\{x \in L^{\infty}\left(\Omega, \mathbb{R}^{m}\right) \mid x(t) \in Q(t) \text { for almost every (a.e.) } t \in \Omega\right\},
$$

where $Q$ is a measurable set-valued map whose images are closed and have nonempty interior, and $(\Omega, \mathcal{A}, \mu)$ is a complete finite measure space. This type of constraint is typical for control constraints in control problems. The main goal of this paper is to investigate this type of constraint and to obtain satisfactory necessary conditions for the corresponding optimization problem.

In the case when $\mathbf{Q}:=\sigma_{\infty}(Q)$, the two questions (i) and (ii) stated above are still open. They can now be rephrased as follows:

$\left(^{*}\right)$ Characterize the critical cone $C\left(x \mid \sigma_{\infty}(Q)\right)$. Furthermore, evaluate the support functional of $V\left(x, d \mid \sigma_{\infty}(Q)\right)$ in terms of the images $Q(t)$ and their support functionals $\delta^{*}(\cdot \mid Q(t))$.

Note that, by [20] and [21], the set $\sigma_{\infty}(Q)$ defined by (1.5) is decomposable, that is,

$$
\chi_{A} x+\chi_{\Omega \backslash A} y \in \sigma_{\infty}(Q) \quad \text { for all } x, y \in \sigma_{\infty}(Q), A \in \mathcal{A} .
$$

(Here $\chi_{A}$ denotes the characteristic function of the set $A$.) Thus, $V\left(x, d \mid \sigma_{\infty}(Q)\right)$ is also decomposable. Therefore, the $L^{1}$-closure of $V\left(x, d \mid \sigma_{\infty}(Q)\right)$ can be identified with a measurable set-valued function $\mathcal{V}: \Omega \rightarrow 2^{\mathbb{R}^{m}}$ whose images are nonempty closed sets.

The aim of this paper is to answer positively the open questions in $\left(^{*}\right)$ when the values of $Q$ are also convex sets. In section 2, the elements of $C\left(x \mid \sigma_{\infty}(Q)\right)$ are characterized in Theorem 2.5 by a certain boundedness condition (2.15). Furthermore, the support function of $V\left(x, d \mid \sigma_{\infty}(Q)\right)$ is evaluated in Theorem 2.2 and Corollaries 2.3 and 2.7 via the evaluation of the support functions associated with the pointwise sets of second-order admissible directions

$$
\mathcal{V}(t):=V(x(t), d(t) \mid Q(t)) \quad(t \in \Omega) .
$$

The results of this section differ from their counterparts established in [19] for the case when $\mathbf{Q}=\sigma_{C}(Q)$ (defined in (1.4)). This distinction is mainly due to the continuity requirement on the selections. In fact, the nonemptiness condition for $V\left(x, d \mid \sigma_{C}(Q)\right.$ in [19] was also phrased in terms of a boundedness below of a lower semicontinuous map (see [19, Theorem 3.5]). On the other hand, the pointwise sets $\mathcal{V}(t)$, defined above, play no role whatsoever in the evaluation of the support function of $V\left(x, d \mid \sigma_{C}(Q)\right)$ (see [19, Theorem 3.10]).

In section 3 , we apply the results of section 2 to the abstract optimization problem $\left(\mathcal{P}^{*}\right)$ (see section 3 ), where two types of parametric constraints are present, namely,

$$
\begin{array}{ll}
g(t, z) \in Q(t) & \text { for a.e. } t \in \Omega, \\
h(t, z)=0 & \text { for a.e. } t \in \Omega,
\end{array}
$$


where $Q$ is as required in (1.5). The main result is given in Theorem 3.1, where the hypotheses and conditions of Theorem 1.1 are phrased in terms of $t$-pointwise conditions. In particular, condition $\left(C_{2}\right)$ is given in terms of the pointwise tangent cone to $Q(t)$ (see condition $\left(C_{2}^{*}\right)$ ) given in Theorem 2.5. The extra term

$$
\delta^{*}\left(x^{*} \mid V\left(G(\hat{z}), G^{\prime}(\hat{z}) y \mid \mathbf{Q}\right)\right)
$$

appearing in the second-order optimality condition is phrased as an integral of a function associated with the set of pointwise second-order admissible variations of $Q(t)$. Another contribution of Theorem 3.1 lies in finding reasonably general conditions $\left(R_{2}^{*}\right)-\left(R_{4}^{*}\right)$ which guarantee that the multipliers associated with the parametric constraints (1.7) are in fact represented via integrable functions.

2. Second-order admissible variations. Let $X=L_{m}^{\infty}:=L^{\infty}\left(\Omega, \mathbb{R}^{m}\right)$, where $(\Omega, \mathcal{A}, \mu)$ is a complete finite measure space, and let $Q: \Omega \rightarrow 2^{\mathbb{R}^{m}}$ be a measurable set-valued function whose images are closed sets with nonempty interior. Define the set $\sigma_{\infty}(Q) \subset L_{m}^{\infty}$ by (1.5). Let $x \in \sigma_{\infty}(Q), d \in L_{m}^{\infty}$, and $\mathbf{V}:=V\left(x, d \mid \sigma_{\infty}(Q)\right)$. In order that $\mathbf{V}$ be nonempty, it is necessary that int $\sigma_{\infty}(Q)$ be nonempty. This latter condition is equivalent (by [21, Theorem 3]; see also [20]) to assuming that $Q$ satisfies

$$
\exists r \geq \rho>0 \text { and, for a.e. } t \in \Omega, \exists x_{t} \in \mathbb{R}^{m} \text { such that } B_{\rho}\left(x_{t}\right) \subset Q(t) \cap B_{r}
$$

(where $B_{\varepsilon}(x)$ denotes the ball in $\mathbb{R}^{m}$ of radius $\varepsilon$ centered at $x$; if $x=0$, then $x$ may be omitted).

A preliminary characterization of $\mathbf{V}$ is given in the following result.

Lemma 2.1. Let $v \in L_{m}^{\infty}$. Then $v \in \mathbf{V}$ if and only if there exist $\bar{\varepsilon}>0$ and a set $A \in \mathcal{A}$ of full measure such that, for all $0<\varepsilon \leq \bar{\varepsilon}, u \in B_{\bar{\varepsilon}} \subset \mathbb{R}^{m}$, and $t \in A$,

$$
x(t)+\varepsilon d(t)+\varepsilon^{2}(v(t)+u) \in Q(t) .
$$

Proof. Let $v \in \mathbf{V}$. Then, by definition, there exists an $\bar{\varepsilon}>0$ such that for all $0<\varepsilon \leq \bar{\varepsilon}, w \in L_{m}^{\infty}$ with $\|w\| \leq \bar{\varepsilon}$, there exists a set $A=A_{\varepsilon, w}$ of full measure that

$$
x(t)+\varepsilon d(t)+\varepsilon^{2}(v(t)+w(t)) \in Q(t) \quad \text { for all } t \in A_{\varepsilon, w} .
$$

Let $\left\{\left(\varepsilon_{n}, u_{n}\right) \mid n \in \mathbb{N}\right\}$ be a dense subset of $[0, \bar{\varepsilon}] \times B_{\bar{\varepsilon}}$. Then defining the measurable functions $w_{n}$ by $w_{n}(t):=u_{n}$, we get from $(2.3)$ that for all $n \in \mathbb{N}$,

$$
x(t)+\varepsilon_{n} d(t)+\varepsilon_{n}^{2}\left(v(t)+u_{n}\right) \in Q(t) \quad \text { for all } t \in \bigcap_{n=1}^{\infty} A_{\varepsilon_{n}, w_{n}} .
$$

Using the fact that $\left\{\left(\varepsilon_{n}, u_{n}\right)\right\}$ is dense and that $Q(t)$ is closed, we obtain that (2.2) is valid for $\varepsilon \leq \bar{\varepsilon}, u \in B_{\bar{\varepsilon}}$, and $t \in A:=\bigcap_{n=1}^{\infty} A_{\varepsilon_{n}, w_{n}}$.

Conversely, let $v \in L_{m}^{\infty}$ and assume that there exists $\bar{\varepsilon}>0$ and $A \in \mathcal{A}$ of full measure such that (2.2) is valid for all $0<\varepsilon \leq \bar{\varepsilon}, u \in B_{\bar{\varepsilon}}$, and $t \in A$. Let $w \in L_{m}^{\infty}$ such that $\|w\| \leq \bar{\varepsilon}$. Then there exists a set $A_{w} \in \mathcal{A}$ of full measure such that $|w(t)| \leq \bar{\varepsilon}$ for all $t \in A_{w}$. Hence, by (2.2), we have (2.3) with $A_{\varepsilon, w}=A \cap A_{w}$. Therefore, $v$ belongs to $\mathbf{V}$.

An immediate consequence of this lemma is that if $v \in \mathbf{V}$, then

$$
v(t) \in V(x(t), d(t) \mid Q(t)) \quad \text { for a.e. } t \in \Omega .
$$


This inclusion motivates the study of the relationship between $\mathbf{V}$ and the measurable set-valued map $\mathcal{V}$ defined by (1.6). Note that the measurability of $\mathcal{V}$ follows from standard arguments.

It is worth noting that (2.4) remains valid when, in $\mathbf{V}, \mathbf{Q}=\sigma_{\infty}(Q)$ is replaced by the set defined in (1.4). In this case, the relationship between $\mathbf{V}$ and $\mathcal{V}$ is not direct, as shown in [20], [21]. However, for the $L^{\infty}$ setting, a direct connection will be established.

We recall now the notions of $L^{1}$-closedness and $L^{1}$-closure from [21]. A subset $\mathbf{Q}$ of $L_{m}^{\infty}$ is called $L^{1}$-closed if whenever $x_{n} \in \mathbf{Q}$ for $n \in \mathbb{N}, x \in L^{\infty}$, and

$$
\lim _{n \rightarrow \infty}\left\|x_{n}-x\right\|_{1}=0
$$

then $x \in \mathbf{Q}$. The $L^{1}$-closure of a set in $L^{\infty}$ is the smallest $L^{1}$-closed set containing it.

Another important type of closedness can be defined in the following way: A subset $\mathbf{Q}$ of $L_{m}^{\infty}$ is called $\Pi$-closed (closed in with respect to the so-called Pontryagin (П-) convergence) if whenever there exists a sequence $x_{n} \in \mathbf{Q}$ such that

$$
\sup \left\|x_{n}\right\|_{\infty}<\infty \text { and } \lim _{n \rightarrow \infty}\left\|x_{n}-x\right\|_{1}=0,
$$

then $x$ has to belong to $\mathbf{Q}$. The $\Pi$-closure of a set is the intersection of all $\Pi$-closed sets containing it. Obviously, the class of $L^{1}$-closed sets forms a proper subclass of the class of $\Pi$-closed sets. However these two notions coincide in the class of decomposable sets as shown by [21, Theorem 1] (see also [20]). The concept of $\Pi$-convergence can also be used to define the notion of the Pontryagin (П-)minimum; see, e.g., [5], [6], [13], [16], [17], and the book by Milyutin and Osmolovskii [15], where necessary and sufficient conditions for this type of minimum are investigated.

Analogously, we can speak about П-continuity of real-valued functions defined on a subset of $L_{m}^{\infty}$, and also about $(\Pi, \Pi)$-continuity of maps from $L_{m}^{\infty}$ to $L_{n}^{\infty}$.

The $L^{1}$-closed and decomposable set $\mathrm{cl}_{1} \mathbf{V}$ is known (by [21, Theorem 2]) to be represented via a measurable set-valued map. As we shall see, this set-valued map is in fact $\overline{\mathcal{V}}$, that is, the set-valued map whose images are $\overline{\mathcal{V}(t)}$ (i.e., the closure of $\mathcal{V}(t)$ ).

ThEOREM 2.2. If $\mathbf{V} \neq \emptyset$, then

$$
\operatorname{cl}_{1} \mathbf{V}=\sigma_{\infty}(\overline{\mathcal{V}})
$$

Proof. The proof of the " $\subset$ " inclusion is obvious since if $v \in \mathbf{V}$, then, from (2.4), we have $v \in \sigma_{\infty}(\mathcal{V}) \subset \sigma_{\infty}(\overline{\mathcal{V}})$. Hence $\mathbf{V} \subset \sigma_{\infty}(\overline{\mathcal{V}})$.

The right-hand side of this inclusion is an $L^{1}$-closed set (see [20], [21]); therefore $\mathrm{cl}_{1} \mathbf{V}$ is also contained in it.

To prove the reversed inclusion in $(2.5)$, assume that $v_{0} \in \sigma_{\infty}(\overline{\mathcal{V}})$. Then, for all $n \in \mathbb{N}$ and for a.e. $t \in \Omega$, the open ball $U_{1 / n}\left(v_{0}(t)\right)$ intersects $\mathcal{V}(t)$. Hence, by known selection theorems for measurable set-valued maps (see [3]), there is a measurable selection $v_{n}$ of the measurable open set-valued map

$$
t \mapsto \mathcal{V}(t) \cap U_{1 / n}\left(v_{0}(t)\right) .
$$

Clearly, $v_{n} \in \sigma_{\infty}(\mathcal{V})$ and $\left\|v_{n}-v_{0}\right\|_{\infty} \leq 1 / n$. Therefore, in order to prove that $v_{0} \in \mathrm{cl}_{1} \mathbf{V}$, it is sufficient to show that $v_{n} \in \mathrm{cl}_{1} \mathbf{V}$. Thus the proof will be completed if we prove

$$
\sigma_{\infty}(\mathcal{V}) \subset \mathrm{cl}_{1} \mathbf{V}
$$


Let $v \in \sigma_{\infty}(\mathcal{V})$. Then there exists a set $A \in \mathcal{A}$ of full measure such that $v(t) \in$ $\mathcal{V}(t)=V(x(t), d(t) \mid Q(t))$ for all $t \in A$. Then, for all $t \in A$, there exists $\bar{\varepsilon}_{t}>0$ such that for all $0<\varepsilon \leq \bar{\varepsilon}_{t}, u \in B_{\bar{\varepsilon}_{t}}$,

$$
x(t)+\varepsilon d(t)+\varepsilon^{2}(v(t)+u) \in Q(t) .
$$

Define, for $(\varepsilon, u) \in(0, \infty) \times \mathbb{R}^{m}$,

$$
A_{\varepsilon, u}:=\left\{t \in A \mid x(t)+\varepsilon d(t)+\varepsilon^{2}(v(t)+u) \in Q(t)\right\} .
$$

Clearly, $A_{\varepsilon, u}$ is measurable. Let $n \in \mathbb{N}$ be fixed, and let $\left\{\left(\varepsilon_{i}, u_{i}\right) \mid i \in \mathbb{N}\right\}$ be a dense subset of $[0,1 / n] \times B_{1 / n}$. Then $\bigcap_{i=1}^{\infty} A_{\varepsilon_{i}, u_{i}}$ is measurable, and by the closedness of $Q(t)$ we have

$$
\begin{aligned}
A_{n} & :=\bigcap_{i=1}^{\infty} A_{\varepsilon_{i}, u_{i}} \\
& =\left\{t \in A \mid x(t)+\varepsilon d(t)+\varepsilon^{2}(v(t)+u) \in Q(t): \text { for all } \varepsilon \in[0,1 / n], \text { for all } u \in B_{1 / n}\right\} .
\end{aligned}
$$

For all $t \in A$, there exists $n \in \mathbb{N}$ such that $\varepsilon_{t}>1 / n$; hence $\bigcup_{n=1}^{\infty} A_{n}=A$. Thus $\mu\left(A_{n}\right) \rightarrow \mu(\Omega)$ as $n \rightarrow \infty$.

Let $\bar{v}$ be a fixed element of $\mathbf{V}$ (which exists by the assumption $\mathbf{V} \neq \emptyset$ ), and define the sequence of functions $\bar{v}_{n}$ by

$$
\bar{v}_{n}(t):= \begin{cases}v(t) & \text { if } t \in A_{n}, \\ \bar{v}(t) & \text { if } t \notin A_{n} .\end{cases}
$$

Since $\bar{v} \in \mathbf{V}$, and using Lemma 2.1, there exist a positive $\bar{\varepsilon}$ and a set $\bar{A} \in \mathcal{A}$ of full measure such that

$$
x(t)+\varepsilon d(t)+\varepsilon^{2}(\bar{v}(t)+u) \in Q(t)
$$

for all $\varepsilon \in[0, \bar{\varepsilon}], u \in B_{\bar{\varepsilon}}$, and $t \in A_{0}$. Taking $\bar{\varepsilon}_{n}=\min (\bar{\varepsilon}, 1 / n)$, we get that

$$
x(t)+\varepsilon d(t)+\varepsilon^{2}\left(\bar{v}_{n}(t)+u\right) \in Q(t)
$$

if $\varepsilon \in\left[0, \bar{\varepsilon}_{n}\right], u \in B_{\bar{\varepsilon}_{n}}$, and $t \in A \cap \bar{A}$. It follows from Lemma 2.1 that $\bar{v}_{n} \in \mathbf{V}$. On the other hand, the sequence $\bar{v}_{n}$ converges to $v$ in the $L^{1}$-norm (since $\mu\left(A_{n}\right) \rightarrow \mu(\Omega)$ as $n \rightarrow \infty)$. Hence, we obtain that $v \in \operatorname{cl}_{1} \mathbf{V}$, which completes the proof.

Remark 2.1. We already know from (2.4) that

$$
\mathbf{V} \subset \sigma_{\infty}(\mathcal{V})
$$

It is natural to investigate whether $\mathbf{V}$ and $\sigma_{\infty}(\mathcal{V})$ are also related through the reverse inclusion. In Theorem 2.2 we have shown that, by using the $L^{1}$-closure of $\mathbf{V}$,

$$
\sigma_{\infty}(\overline{\mathcal{V}}) \subset \operatorname{cl}_{1}(\mathbf{V})
$$

However, one may ask whether another relation of this type exists by using the $L_{\infty^{-}}$ closure of $\mathbf{V}$. As we shall show in the example below, (2.5) is the only possible such connection. In fact, we shall show that for this example

$$
\sigma_{\infty}(\mathcal{V}) \not \subset \mathrm{cl}_{\infty} \mathbf{V}
$$


and thus also $\sigma_{\infty}(\overline{\mathcal{V}}) \not \subset \mathrm{cl}_{\infty} \mathbf{V}$. Hence (2.5) fails to hold when instead of the $L^{1}$-closure we use the $L^{\infty}$-closure.

Example 2.1. For $t \in \Omega:=(0,1]$, let

$$
Q(t):=\left\{\left(x_{1}, x_{2}\right) \in \mathbb{R}^{2} \mid 0 \leq x_{2}, x_{1} \leq \sqrt{t x_{2}}\right\}
$$

Then the values of $Q$ are closed convex sets; furthermore, $\mathbf{Q}=\sigma_{\infty}(Q)$ has nonempty interior because, for all $t \in \Omega$,

$$
\left\{\left(x_{1}, x_{2}\right) \in \mathbb{R}^{2} \mid x_{1} \leq 0, x_{2} \geq 0\right\} \subset Q(t) .
$$

It is obvious that $x \equiv 0 \in \mathbf{Q}$. Define $d \in L^{\infty}(\Omega)$ by $d(t)=(\sqrt{t}, 0)$. Then, $d \in T(0 \mid \mathbf{Q})$, since with

$$
d_{n}(t):=\left(\sqrt{t}, \varepsilon_{n}\right) \quad(t \in \Omega, n \in \mathbb{N})
$$

(where $\varepsilon_{n} \rightarrow 0+$ ), we have that

$$
\left\|d-d_{n}\right\|_{\infty}=\varepsilon_{n} \quad \text { and } \quad x(t)+\varepsilon_{n} d_{n}(t)=\left(\varepsilon_{n} \sqrt{t}, \varepsilon_{n}^{2}\right) \in Q(t) \quad(t \in \Omega, n \in \mathbb{N}) .
$$

Now we show that, for all $t \in \Omega$,

$$
V(x(t), d(t) \mid Q(t))=\left\{\left(v_{1}, v_{2}\right) \in \mathbb{R}^{2} \mid v_{2}>1\right\} .
$$

Let $t \in \Omega$ be fixed. Then $\left(v_{1}, v_{2}\right)$ belongs to $V(x(t), d(t) \mid Q(t))$ if and only if there exists $\bar{\varepsilon}>0$ such that, for all $0<\varepsilon<\bar{\varepsilon},\left|\left(u_{1}, u_{2}\right)\right|<\bar{\varepsilon}$,

$$
\left(\varepsilon \sqrt{t}+\varepsilon^{2}\left(v_{1}+u_{1}\right), \varepsilon^{2}\left(v_{2}+u_{2}\right)\right) \in Q(t),
$$

that is,

$$
0 \leq v_{2}+u_{2} \quad \text { and } \quad 1+\frac{\varepsilon\left(v_{1}+u_{1}\right)}{\sqrt{t}} \leq \sqrt{v_{2}+u_{2}} .
$$

Taking the limit $\varepsilon \rightarrow 0$, it follows that $v_{2}+u_{2} \geq 1$ if $\left|u_{2}\right|<\bar{\varepsilon}$. Hence $v_{2}>1$ is a necessary condition.

Conversely, if $v_{2}>1$ and $v_{1} \in \mathbb{R}$, then there exists a constant $c>0$ such that

$$
1 \leq v_{2}-c, \quad 1+c\left(v_{1}+c\right) \leq \sqrt{v_{2}-c} .
$$

Then, for $\left|u_{i}\right| \leq c$, we get

$$
1 \leq \sqrt{v_{2}+u_{2}}, \quad 1+c\left(v_{1}+u_{1}\right) \leq \sqrt{v_{2}+u_{2}} .
$$

Multiplying the first inequality by $1-\varepsilon /(c \sqrt{t})$, the second by $\varepsilon /(c \sqrt{t})$, and adding the two inequalities so obtained, we get that (2.7) holds for $0<\varepsilon \leq c \sqrt{t},\left|u_{1}\right| \leq c$, $\left|u_{2}\right| \leq c$. Hence, $\left(v_{1}, v_{2}\right) \in V(x(t), d(t) \mid Q(t))$.

Thus, all the constant functions $v(t)=\left(v_{1}, v_{2}\right)$, where $v_{2}>1$, belong to $\sigma_{\infty}(\mathcal{V})$. Now, we prove that $v \notin \mathrm{cl}_{\infty} \mathbf{V}$ for $v_{1}>0$.

We argue by contradiction. Assume that $v_{1}>0, v_{2}>1$, and $v \equiv\left(v_{1}, v_{2}\right) \in \mathrm{cl}_{\infty} \mathbf{V}$. Then there exists a sequence $w_{n} \in \mathbf{V}$ with $\left\|w_{n}-v\right\|_{\infty} \rightarrow 0$. By Lemma 2.1, $w_{n} \in \mathbf{V}$ means that there exist $0<\varepsilon_{n} \leq 1$ and $A_{n} \subset \Omega$ of full measure such that, for all $0<\varepsilon \leq \varepsilon_{n}, u \in B_{\varepsilon_{n}} \subset \mathbb{R}^{2}$, and $t \in A_{n}$,

$$
0 \leq w_{n, 2}(t)+u_{2}, \quad 1+\frac{\varepsilon}{\sqrt{t}}\left(w_{n, 1}(t)+u_{1}\right) \leq \sqrt{w_{n, 2}(t)+u_{2}} .
$$


Hence, by taking $u_{1}=u_{2}=0$ and $\varepsilon=\varepsilon_{n}$, we have for all $n \in \mathbb{N}$ and $t \in A:=\bigcap_{n=1}^{\infty} A_{n}$,

$$
1+\frac{\varepsilon_{n}}{\sqrt{t}} w_{n, 1}(t) \leq \sqrt{w_{n, 2}(t)}
$$

Thus, due to the $L^{\infty}$-convergence of $w_{n}$ to $v \equiv\left(v_{1}, v_{2}\right)$, we have for some $n_{0} \in \mathbb{N}$ and $\bar{A} \subset \Omega$ of full measure that $w_{n, 1}(t)>v_{1} / 2$ and $w_{n, 2}(t)<2 v_{2}$ if $t \in \bar{A}$ and $n \geq n_{0}$. Hence,

$$
1+\frac{\varepsilon_{n} v_{1}}{2 \sqrt{t}} \leq \sqrt{2 v_{2}}, \quad \text { that is, } \quad \varepsilon_{n} \leq 2 \sqrt{t} \frac{\sqrt{2 v_{2}}-1}{v_{1}}
$$

for $t \in A \cap \bar{A}$ and for $n \geq n_{0}$. Therefore, $\varepsilon_{n}=0$ for $n \geq n_{0}$, contradicting $\varepsilon_{n}>0$.

It is worth noting that in the above example the boundary of the set $Q(t)$ has at $(0,0)$ a curvature of order $1 / t$, and hence it is not bounded above on $\Omega$.

An essential result follows from Theorem 2.2 that shows how to express the support function of $\mathbf{V}$ in terms of that of $\mathcal{V}(t)$.

Corollary 2.3. Assume that $\mathbf{V} \neq \emptyset$. Then, for $\varphi \in L_{m}^{1}$,

$$
\delta^{*}(\varphi \mid \mathbf{V})=\int_{\Omega} \delta^{*}(\varphi(t) \mid \mathcal{V}(t)) d \mu(t)
$$

Proof. Applying the previous theorem and [21, Lemma 4 and Theorem 6], which employ results from [25], [26] and [9], we have that

$$
\begin{aligned}
\delta^{*}(\varphi \mid \mathbf{V}) & =\delta^{*}\left(\varphi \mid \mathrm{cl}_{1} \mathbf{V}\right)=\delta^{*}\left(\varphi \mid \sigma_{\infty}(\overline{\mathcal{V}})\right) \\
& =\int_{\Omega} \delta^{*}(\varphi(t) \mid \overline{\mathcal{V}}(t)) d \mu(t)=\int_{\Omega} \delta^{*}(\varphi(t) \mid \mathcal{V}(t)) d \mu(t) .
\end{aligned}
$$

The above results bring up the questions of studying

(i) the characterization of the nonemptiness of $\mathbf{V}$, and

(ii) the calculation of the support function of the images $\mathcal{V}(t)$.

If the images of the set-valued map $Q$ are convex, then $\sigma_{\infty}(Q)$ is also convex. In this case, Theorem 2.5 below offers an important characterization for the nonemptiness of $\mathbf{V}$. This result is based on the following characterization of the elements of $\mathbf{V}$ that is more useful in this case than that given by Lemma 2.1.

To state these results concisely, we introduce the following functions. For $t \in \Omega$ and $\xi \in \mathbb{R}^{m}$, denote

$$
a(t, \xi):=\delta^{*}(\xi \mid Q(t))-\langle\xi, x(t)\rangle, \quad b(t, \xi):=\langle\xi, d(t)\rangle .
$$

Lemma 2.4. Let $x \in \sigma_{\infty}(Q)$ and $d \in L_{m}^{\infty} . v \in \mathbf{V}=V\left(x, d \mid \sigma_{\infty}(Q)\right)$ if and only if

$$
d(t) \in T(x(t) \mid Q(t))
$$

for a.e. $t \in \Omega$, and there exists $\bar{\varepsilon}>0$ such that, for a.e. $t \in \Omega$,

$$
\langle\xi, v(t)\rangle \leq \begin{cases}-\bar{\varepsilon}|\xi|-\frac{[b(t, \xi)]^{2}}{4 a(t, \xi)} & \text { if } \bar{\varepsilon} b(t, \xi)>2 a(t, \xi), \\ -\bar{\varepsilon}|\xi|+\frac{a(t, \xi)-\bar{\varepsilon} b(t, \xi)}{\bar{\varepsilon}^{2}} & \text { if } \bar{\varepsilon} b(t, \xi) \leq 2 a(t, \xi)\end{cases}
$$

for all $\xi \in \mathbb{R}^{m} \backslash\{0\}$. 
Proof. Let $v \in \mathbf{V}$. Then $\mathbf{V}$ is nonempty; hence $d \in T(x \mid \mathbf{Q})$. Then, it follows from [21, Theorem 4] that (2.10) is valid for a.e. $t \in \Omega$.

To prove (2.11), note that for almost all $t \in \Omega$, in the first domain, that is, in the set

$$
\left\{\xi \in \mathbb{R}^{m} \mid \bar{\varepsilon} b(t, \xi)>2 a(t, \xi)\right\},
$$

we have $a(t, \xi)>0$. This implies that the function defined in the right-hand side of the above inequality is well defined. Indeed, by (2.10), the equality $a(t, \xi)=$ $\delta^{*}\left(\xi \mid \sigma_{\infty}(Q)\right)-\langle\xi, x(t)\rangle=0$ yields $b(t, \xi)=0$, contradicting $\bar{\varepsilon} b(t, \xi)>2 a(t, \xi)=0$.

By Lemma 2.1, there exist $\bar{\varepsilon}>0$ and a set $A \in \mathcal{A}$ of full measure such that (2.2) holds for all $0<\varepsilon \leq \bar{\varepsilon}, u \in B_{\bar{\varepsilon}} \subset \mathbb{R}^{m}$, and $t \in A$. Therefore, for all $\xi \in \mathbb{R}^{m}$, we have

$$
\langle\xi, x(t)\rangle+\varepsilon\langle\xi, d(t)\rangle+\varepsilon^{2}\langle\xi, v(t)+u\rangle \leq \delta^{*}(\xi \mid Q(t)),
$$

that is,

$$
\varepsilon b(t, \xi)+\varepsilon^{2}\langle\xi, v(t)+u\rangle \leq a(t, \xi) .
$$

Putting $u=\bar{\varepsilon} \xi /|\xi|$, we deduce

$$
\varepsilon b(t, \xi)+\varepsilon^{2}(\langle\xi, v(t)\rangle+\bar{\varepsilon}|\xi|) \leq a(t, \xi)
$$

for all $0<\varepsilon \leq \bar{\varepsilon}, t \in A$, and $\xi \in \mathbb{R}^{m} \backslash\{0\}$. Hence,

$$
\langle\xi, v(t)\rangle \leq-\bar{\varepsilon}|\xi|+\inf _{0<\varepsilon \leq \bar{\varepsilon}} \frac{a(t, \xi)-\varepsilon b(t, \xi)}{\varepsilon^{2}} .
$$

Computing the infimum on the right-hand side, we get that (2.11) is valid for all $t \in A$ and $\xi \in \mathbb{R}^{m} \backslash\{0\}$.

Conversely, if $v \in L_{m}^{\infty},(2.10)$ is valid, and there exists $\bar{\varepsilon}>0$ and a set $A \in \mathcal{A}$ of full measure such that $v$ satisfies (2.11) for all $\xi \in\left(\mathbb{R}^{m} \backslash\{0\}\right)$, then (2.14) and (2.13) are also valid. Thus (2.12) holds for all $u \in B_{\bar{\varepsilon}}$. The set $Q(t)$ being convex, this latter inequality implies (2.2). Hence, by Lemma 2.1 again, $v$ belongs to $\mathbf{V}$.

Thus we have proved Lemma 2.4 .

Remark 2.2. An interesting consequence of Lemma 2.4 is that if $(2.10)$ is valid and (2.11) also holds on the domain indicated, then $d \in T\left(x \mid \sigma_{\infty}(Q)\right)$. The condition (2.10) alone is only a necessary condition for $d$ to be in the tangent cone of $\sigma_{\infty}(Q)$ at $x$ (see [21]). If the images of $Q$ are not convex, then (2.10) and (2.11) are only necessary for $v$ to be in $\mathbf{V}$.

ThEOREM 2.5. Let $Q: \Omega \rightarrow 2^{\mathbb{R}^{m}}$ be a measurable set-valued map whose images are closed convex sets and satisfy (2.1). Let $x \in \sigma_{\infty}(Q)$ and let $d \in L_{m}^{\infty}$. Then the set of second-order admissible variations $\mathbf{V}=V\left(x, d \mid \sigma_{\infty}(Q)\right)$ is nonempty if and only if there exists a constant $M>0$ such that, for a.e. $t \in \Omega$, the following condition is valid:

$$
[b(t, \xi)]^{2} \leq M|\xi| a(t, \xi) \quad \text { whenever } \quad \xi \in \mathbb{R}^{m} \text { and } b(t, \xi)>0
$$

(where the functions $a$ and $b$ are defined in (2.9)).

Remark 2.3. From Theorem 2.5 it readily follows that, for a.e. $t, \mathcal{V}(t)=$ $V(x(t), d(t) \mid Q(t))$ is nonempty (that is, $d(t) \in C(x(t) \mid Q(t))$ ) if and only if (2.15) 
holds for some $M_{t}>0$ on the domain indicated. Therefore, Theorem 2.5 can be rephrased as

$d \in C\left(x \mid \sigma_{\infty}(Q)\right) \Longleftrightarrow\left\{\begin{array}{l}d(t) \in C(x(t) \mid Q(t)) \text { for a.e. } t \in \Omega \text { uniformly, i.e., } \\ \exists M_{t}, \exists M>0: M_{t} \text { satisfies }(2.15), M_{t} \leq M \text { for a.e. } t \in \Omega .\end{array}\right.$

Proof. Assume that $v \in \mathbf{V}$. Then, by Lemma 2.4, there exist $\bar{\varepsilon}>0$, a set $A \in \mathcal{A}$ of full measure such that (2.11) is true for all $t \in A$ and $\xi \in \mathbb{R}^{m} \backslash\{0\}$.

Let $t \in A$ and $\xi \in \mathbb{R}^{m}$ such that $b(t, \xi)>0$. Then, as we have seen in the proof of Lemma 2.4, $a(t, \xi)>0$. We distinguish two cases: If $\bar{\varepsilon} b(t, \xi)>2 a(t, \xi)>0$, then, by the first inequality in (2.11), we have

$$
\frac{[b(t, \xi)]^{2}}{a(t, \xi)} \leq-4\langle\xi, v(t)\rangle-4 \bar{\varepsilon}|\xi| \leq 4|\xi|(\|v\|-\bar{\varepsilon}) .
$$

In the other case, i.e., if $\bar{\varepsilon} b(t, \xi) \leq 2 a(t, \xi)$ is valid, we have

$$
\frac{[b(t, \xi)]^{2}}{a(t, \xi)} \leq \frac{2 b(t, \xi)}{\bar{\varepsilon}}=\frac{2\langle\xi, d(t)\rangle}{\bar{\varepsilon}} \leq \frac{2|\xi|\|d\|}{\bar{\varepsilon}} .
$$

Hence with the constant $M$ defined as

$$
M:=\max \left(4(\|v\|-\bar{\varepsilon}), \frac{2\|d\|}{\bar{\varepsilon}}\right)
$$

we get that (2.15) holds on the indicated domain.

Conversely, assume that (2.15) is valid. Then (2.10) holds, because, if $d(t) \notin$ $T(x(t) \mid Q(t))$, then there exists $\xi \in N(x(t) \mid Q(t))$ such that $\langle\xi, d(t)\rangle>0$. This yields that $a(t, \xi)=0$ and, by $(2.15), b(t, \xi)=\langle\xi, d(t)\rangle=0$, leading to a contradiction. by

$$
v=\frac{w-x-\bar{\varepsilon} d}{\bar{\varepsilon}^{2}}
$$

belongs to $\mathbf{V}$.

As we have noted above, by [21, Theorem 3], the condition in (2.1) yields that $\sigma_{\infty}(Q)$ has nonempty interior. Moreover the centers $x_{t}$ in $(2.1)$ can be chosen in a measurable way. Define the measurable function $w$ by $w(t)=x_{t}$. Then, from (2.1) it results that there exists a set $A$ of full measure such that, for all $t \in A$, we have that $w(t)+B_{\rho} \subset Q(t)$. Hence,

$$
\langle\xi, w(t)\rangle+\rho|\xi| \leq \delta^{*}(\xi \mid Q(t)) \quad \text { for all } t \in A, \xi \in \mathbb{R}^{m} .
$$

Let $M$ be the constant that validates (2.15). Choose $\bar{\varepsilon}>0$ so that $\bar{\varepsilon}^{2}(\bar{\varepsilon}+M / 4) \leq \rho$. Thus, we have

$$
\langle\xi, w(t)\rangle+\bar{\varepsilon}^{2}(\bar{\varepsilon}+M / 4)|\xi| \leq \delta^{*}(\xi \mid Q(t)) \quad \text { for all } t \in A, \xi \in \mathbb{R}^{m} .
$$

To complete the proof of the theorem, we need to show that the function $v$ defined in (2.16) satisfies (2.11) with this $\bar{\varepsilon}$.

Substituting $v=(w-x-\bar{\varepsilon} d) / \bar{\varepsilon}^{2}$ into this condition, it remains to prove that

$$
\langle\xi, w(t)\rangle \leq \begin{cases}-\bar{\varepsilon}^{3}|\xi|-\bar{\varepsilon}^{2} \frac{[b(t, \xi)]^{2}}{4 a(t, \xi)}+\langle\xi, x(t)\rangle+\bar{\varepsilon} b(t, \xi) & \text { if } \bar{\varepsilon} b(t, \xi)>2 a(t, \xi), \\ -\bar{\varepsilon}^{3}|\xi|+\delta^{*}(\xi \mid Q(t)) & \text { if } \bar{\varepsilon} b(t, \xi) \leq 2 a(t, \xi)\end{cases}
$$


for all $t \in A, \xi \in\left(\mathbb{R}^{m} \backslash\{0\}\right)$.

By the choice of $\bar{\varepsilon}$, we have that $\bar{\varepsilon}^{3} \leq \rho$, and hence

$$
\langle\xi, w(t)\rangle+\bar{\varepsilon}^{3}|\xi| \leq \delta^{*}(\xi \mid Q(t)) \quad \text { for all } t \in A, \xi \in \mathbb{R}^{m},
$$

that is, the second inequality above holds.

It remains to show that the first inequality holds on its domain.

If $(t, \xi)$ belongs to this domain, then

$$
\bar{\varepsilon} b(t, \xi)>2 a(t, \xi)>a(t, \xi)>0 .
$$

Thus, by our assumption, there exists a positive constant $M$ such that (2.15) is valid. Combining these inequalities, we obtain

$$
\begin{aligned}
\langle\xi, w(t)\rangle & \leq \delta^{*}(\xi \mid Q(t))-\bar{\varepsilon}^{2}(\bar{\varepsilon}+M / 4)|\xi| \\
& =a(t, \xi)+\langle\xi, x(t)\rangle-\bar{\varepsilon}^{2}(\bar{\varepsilon}+M / 4)|\xi| \\
& <\bar{\varepsilon} b(t, \xi)+\langle\xi, x(t)\rangle-\bar{\varepsilon}^{3}|\xi|-\bar{\varepsilon}^{2} M|\xi| / 4 \\
& \leq \bar{\varepsilon} b(t, \xi)+\langle\xi, x(t)\rangle-\bar{\varepsilon}^{3}|\xi|-\bar{\varepsilon}^{2} \frac{[b(t, \xi)]^{2}}{4 a(t, \xi)}
\end{aligned}
$$

for all $(t, \xi)$ satisfying $\bar{\varepsilon} b(t, \xi)>2 a(t, \xi)$, that is, the needed first inequality above is proved, and hence $v$ is in $\mathbf{V}$. Thus the nonemptiness of $\mathbf{V}$ is proved and the proof of Theorem 2.5 is complete.

Remark 2.4. It follows from Theorem 2.5 that if there exists a constant $M$ such that (2.15) is satisfied on the domain indicated, then $d \in T\left(x \mid \sigma_{\infty}(Q)\right)$.

The rest of this section is devoted to answering the question pertaining the calculation of the support function of the images of $\mathcal{V}(t)$ in terms of $x(t), d(t)$, and $Q(t)$. Thus, for fixed $t$, we need to calculate $\delta^{*}(\xi \mid V(x(t), d(t) \mid Q(t)))$. For this reason, we recall a special case of the result derived in [22] that describes the set $V(x, d \mid Q)$, for $x \in \mathbb{R}^{m}, d \in T(x \mid Q)$, and $Q$ a convex set in $\mathbb{R}^{m}$ with nonempty interior, in terms of its support functional.

Denote

$$
d^{\perp}:=\left\{\xi \in \mathbb{R}^{m} \mid\langle\xi, d\rangle=0\right\}, \quad d^{>}:=\left\{\xi \in \mathbb{R}^{m} \mid\langle\xi, d\rangle>0\right\},
$$

and define from $\mathbb{R}^{m}$ to the extended reals the function

$$
\sigma(x, d \mid Q)(\xi):= \begin{cases}\liminf _{\zeta \rightarrow \xi} \frac{[\langle\zeta, d\rangle]^{2}}{4\left[\langle\zeta, x\rangle-\delta^{*}(\zeta \mid Q)\right]} & \text { if } \xi \in N(x \mid Q) \cap d^{\perp} \\ \zeta \in d^{\zeta} & \text { otherwise. }\end{cases}
$$

One can see that $\sigma(x, d \mid Q)(\cdot)$ is a positively homogeneous function and also lower semicontinuous on $\mathbb{R}^{m} \backslash\{0\}$.

Define the convex regularization $\overline{\mathrm{co}} \sigma(x, d \mid Q)(\cdot)$ to be the largest lower semicontinuous convex function below $\sigma(x, d \mid Q)$, that is,

$$
\begin{gathered}
\overline{\operatorname{co}} \sigma(x, d \mid Q)(\xi)=\sup \left\{\varphi(\xi) \mid \varphi: \mathbb{R}^{m} \rightarrow[-\infty, \infty]\right. \text { is convex and lower semicontinuous, } \\
\left.\varphi(\zeta) \leq \sigma(x, d \mid Q)(\zeta) \text { for all } \zeta \in \mathbb{R}^{m} \backslash\{0\}\right\} .
\end{gathered}
$$

It results that $\overline{\operatorname{co}} \sigma(x, d \mid Q)(\cdot)$ is also sublinear. 
TheOREm 2.6. Let $Q \subset \mathbb{R}^{m}$ be closed convex with nonempty interior, let $x \in Q$, $d \in C(x \mid Q)$. Then a vector $v \in \mathbb{R}^{m}$ belongs to $V(x, d \mid Q)$ if and only if

$$
\langle\xi, v\rangle<\overline{\operatorname{co}} \sigma(x, d \mid Q)(\xi) \quad \text { for all } \xi \in \mathbb{R}^{m} \backslash\{0\} .
$$

Furthermore, for all $\xi \in \mathbb{R}^{m}$,

$$
\delta^{*}(\xi \mid V(x, d \mid Q))=\overline{\operatorname{co}} \sigma(x, d \mid Q)(\xi) .
$$

The following result offers an evaluation of the support function of the set $\mathbf{V}=$ $V\left(x, d \mid \sigma_{\infty}(Q)\right)$ at linear functionals that can be represented in terms of integrable functions.

Corollary 2.7. Let $Q$ be a measurable set-valued map on $\Omega$ whose images are closed convex sets that satisfy condition (2.1), let $x \in \sigma_{\infty}(Q)$ and $d \in C\left(x, d \mid \sigma_{\infty}(Q)\right)$, and let $\varphi \in L^{1}\left(\Omega, \mathbb{R}^{m}\right)$. Then

$$
\delta^{*}(\varphi \mid \mathbf{V})=\int_{\Omega} \overline{\operatorname{co}} \sigma(x(t), d(t) \mid Q(t))(\varphi(t)) d \mu(t) .
$$

3. Applications to optimization theory. In this section we make a specification of the optimization problem $(\mathcal{P})$ and Theorem 1.1. Let $Y, Z$ be Banach spaces, $\mathcal{D} \subset Z$ nonempty and open, $F_{i}: \mathcal{D} \rightarrow \mathbb{R}(i=0, \ldots, p)$, and $K: \mathcal{D} \rightarrow Y$ be given. Let $(\Omega, \mathcal{A}, \mu)$ be a finite complete measure space, $g: \Omega \times \mathcal{D} \rightarrow \mathbb{R}^{m}, h: \Omega \times \mathcal{D} \rightarrow \mathbb{R}^{n}$, and $Q: \Omega \rightarrow 2^{\mathbb{R}^{m}}$ be a measurable set-valued map whose values are closed convex sets and the condition (2.1) is satisfied. Then, as stated in the previous section, $\sigma_{\infty}(Q) \subset L_{m}^{\infty}$ has nonempty interior.

We consider the following optimization problem:

$\left(\mathcal{P}^{*}\right) \quad$ Minimize $\quad F_{0}(z)$ subject to $\quad\left\{\begin{array}{l}F(z) \leq 0, \\ g(t, z) \in Q(t) \text { for a.e. } t \in \Omega, \\ h(t, z)=0 \text { for a.e. } t \in \Omega, \\ K(z)=0 .\end{array}\right.$

Introduce the functions $H: \mathcal{D} \rightarrow L_{n}^{\infty}$ and $G: \mathcal{D} \rightarrow L_{m}^{\infty}$ by

$$
H(z)(t)=h(t, z) \quad \text { and } \quad G(z)(t)=g(t, z) .
$$

Then, with $E:=(H, K)$ and $F:=\left(F_{1}, \ldots, F_{p}\right)$, the problem $\left(\mathcal{P}^{*}\right)$ reduces to $(\mathcal{P})$ described in the introduction.

The main focus of this section is to apply Theorem 1.1 to the problem $\left(\mathcal{P}^{*}\right)$ in such a way that all of the hypotheses assumed and all the results obtained will be phrased explicitly in terms of the data $F_{0}, F, g, Q, h$, and $K$.

Now we define the notions of a solution, admissible and regular points, and critical and regular directions.

- A point $\hat{z} \in \mathcal{D}$ is admissible for $\left(\mathcal{P}^{*}\right)$ if $F(\hat{z}) \leq 0, g(t, \hat{z}) \in Q(t), h(t, \hat{z})=0$ for a.e. $t \in \Omega$ and $K(\hat{z})=0$. A point $\hat{z} \in \mathcal{D}$ is a solution (local minimum) for this problem if there exists a neighborhood $U$ of $\hat{z}$ such that $F_{0}(z) \geq F_{0}(\hat{z})$ for all admissible point $z \in U$.

- The regularity of an admissible solution $\hat{z}$ means that the assumption $\left(R_{1}\right)$ is valid and, in addition, we have $\left(R_{1}^{*}\right)-\left(R_{4}^{*}\right)$ below.

$\left(R_{1}^{*}\right)$ The map $l(t, \cdot):=(g, h)(t, \cdot)$ is $L^{\infty}$-uniformly strictly Fréchet differentiable at $\hat{z}$ for a.e. $t \in \Omega$, that is,

$$
\lim _{z_{1}, z_{2} \rightarrow \hat{z}} \frac{\left|l\left(t, z_{1}\right)-l\left(t, z_{2}\right)-l^{\prime}(t, \hat{z})\left(z_{1}-z_{2}\right)\right|}{\left\|z_{1}-z_{2}\right\|}=0
$$


holds $L^{\infty}$-uniformly for $t \in \Omega$. (Then the maps $G, H$ defined by (3.1) are strictly Fréchet differentiable at $\hat{z}$.)

$\left(R_{2}^{*}\right)$ There exist a mapping $A: L_{m}^{\infty} \rightarrow Z$ and a bounded linear operator $B: L_{n}^{\infty} \rightarrow Z$ such that $A(0)=0$,

$$
H^{\prime}(\hat{z}) B=I, \quad H^{\prime}(\hat{z}) \circ A=0,
$$

and

$$
G^{\prime}(\hat{z})(A(w))-w \in T\left(G(\hat{z}) \mid \sigma_{\infty}(Q)\right) \quad \text { for all } \quad w \in L_{m}^{\infty} .
$$

Moreover, the operator $G^{\prime}(\hat{z}) \circ B$ is a $(\Pi, \Pi)$-continuous map at zero from $L_{n}^{\infty}$ to $L_{m}^{\infty}$.

$\left(R_{3}^{*}\right) F_{0}$ and $F$ are locally Lipschitz at $\hat{z}$ and the functions $F_{i}^{\circ}(\hat{z}, A(\cdot))$ and $F_{i}^{\circ}(\hat{z}, B(\cdot))$ are $\Pi$-continuous at zero for all $i=0, \ldots, p$.

$\left(R_{4}^{*}\right) K$ is strictly Fréchet differentiable at $\hat{z}, K^{\prime}(\hat{z}) \circ A$ and $K^{\prime}(\hat{z}) \circ B$ are weakly $\Pi$-continuous, and $K^{\prime}(\hat{z}) \circ\left(I_{Z}-B \circ H^{\prime}(\hat{z})\right): Z \rightarrow Y$ has a closed range (where $I_{Z}$ is the identity on $Z$ ).

- A direction $y \in Z$ is critical for $\left(\mathcal{P}^{*}\right)$ at an admissible regular point $\hat{z}$ if $\left(C_{1}\right)$ is valid, and

$\left(C_{2}^{*}\right) g^{\prime}(t, \hat{z})(y) \in C(g(t, \hat{z}) \mid Q(t))$ for a.e. $t \in \Omega$ uniformly, that is, there exists a constant $M$ such that for a.e. $t \in \Omega$,

$$
\left[\left\langle\xi, g^{\prime}(t, \hat{z})(y)\right\rangle\right]^{2} \leq M|\xi|\left(\delta^{*}(\xi \mid Q(t))-\langle\xi, g(t, \hat{z})\rangle\right)
$$

for all $\xi \in \mathbb{R}^{m}$ such that $\left\langle\xi, g^{\prime}(t, \hat{z})(y)\right\rangle>0$.

$\left(C_{3}^{*}\right) h^{\prime}(t, \hat{z})(y)=0$ for a.e. $t \in \Omega$ and $K^{\prime}(\hat{z})(y)=0$.

- The vector $y$ is a regular direction if $\left(R_{4}\right)$ and the following hold:

$\left(R_{5}^{*}\right)$ The following second-order directional derivative exists for the function $l:=(g, h)$ for a.e. $t \in \Omega$,

$$
l^{\prime \prime}(t, \hat{z}, y):=\lim _{\varepsilon \rightarrow 0+} 2 \frac{l(t, \hat{z}+\varepsilon y)-l(t, \hat{z})-\varepsilon l^{\prime}(t, \hat{z}) y}{\varepsilon^{2}},
$$

and the limit is $L^{\infty}$-uniform in $t$; furthermore, $K$ satisfies the same assumption as the function $E$ in (R5).

The main result of the section is the following theorem. Its proof employs the results derived in section 2 and Theorem 1.1.

THEOREM 3.1. Let $\hat{z}$ be a regular solution of the above problem $\left(\mathcal{P}^{*}\right)$. Then, for all regular critical directions $y$, there correspond Lagrange multipliers $\lambda_{0}, \lambda_{1}, \ldots, \lambda_{p} \geq 0$, $\varphi \in L_{m}^{1}, \psi \in L_{n}^{1}$, and $y^{*} \in Y^{*}$ (depending on $y$ ) that do not vanish simultaneously, and the following relations hold:

$$
\lambda_{i} F_{i}(\hat{z})=0(i=1, \ldots, p), \quad \lambda_{i} F_{i}^{o}(\hat{z} ; y)=0(i=0, \ldots, p)
$$

for a.e. $t \in \Omega$,

$$
\begin{gathered}
\varphi(t) \in N(g(t, \hat{z}) \mid Q(t)), \quad\left\langle\varphi(t), g^{\prime}(t, \hat{z})(y)\right\rangle=0, \\
\sum_{i=0}^{p} \lambda_{i} F_{i}^{o}(\hat{z} ; z)+\left\langle y^{*}, K^{\prime}(\hat{z})(z)\right\rangle \\
+\int_{\Omega}\left[\left\langle\varphi(t), g^{\prime}(t, \hat{z})(z)\right\rangle+\left\langle\psi(t), h^{\prime}(t, \hat{z})(z)\right\rangle\right] d \mu(t) \geq 0 \quad \text { for } z \in Z,
\end{gathered}
$$


and

$$
\begin{aligned}
& \sum_{i=0}^{p} \lambda_{i} F_{i}^{o \prime}(\hat{z} ; y)+\left\langle y^{*}, K^{\prime \prime}(\hat{z})(y)\right\rangle \\
& +\int_{\Omega}\left[\left\langle\varphi(t), g^{\prime \prime}(t, \hat{z})(y)\right\rangle+\left\langle\psi(t), h^{\prime \prime}(t, \hat{z})(y)\right\rangle\right] d \mu(t), \geq 2 \int_{\Omega} \gamma(t, \varphi(t)) d \mu(t)
\end{aligned}
$$

where $\gamma(t, \xi):=\overline{\operatorname{co}} \sigma\left(g(t, \hat{z}), g^{\prime}(t, \hat{z}) y \mid Q(t)\right)(\xi)$.

Observe that, using the Hahn-Banach theorem, the first-order condition (3.4) can be written as an equality: There exist linear functionals $z_{i}^{*} \in \partial F_{i}(\hat{z})(i=0, \ldots, p)$ such that

$$
\begin{aligned}
& \sum_{i=0}^{p} \lambda_{i}\left\langle z_{i}^{*}, z\right\rangle+\left\langle y^{*}, K^{\prime}(\hat{z})(z)\right\rangle \\
& +\int_{\Omega}\left[\left\langle\varphi(t), g^{\prime}(t, \hat{z})(z)\right\rangle+\left\langle\psi(t), h^{\prime}(t, \hat{z})(z)\right\rangle\right] d \mu(t)=0 \quad \text { for } z \in Z .
\end{aligned}
$$

Proof. We intend to apply Theorem 1.1 to our problem $\left(\mathcal{P}^{*}\right)$. First we verify that all the hypotheses of Theorem 1.1 concerning $\hat{z}$ and the critical direction $y$ are satisfied.

From $\left(R_{1}^{*}\right)$, it follows that $G$ and $H$ are strictly Fréchet differentiable at $\hat{z}$. Thus, $\left(R_{1}\right)-\left(R_{3}\right)$ will be satisfied if we show that, for $E:=(H, K)$, the operator $E^{\prime}(\hat{z})$ has a closed range in $L_{n}^{\infty} \times Y$.

From $\left(R_{2}^{*}\right)$ we have the surjectivity of $H^{\prime}(\hat{z})$. Then, by [1, Lemma 2.1.6], the result follows if we show that $K^{\prime}(\hat{z})\left(\operatorname{Ker} H^{\prime}(\hat{z})\right)$ is a closed subspace. By $\left(R_{2}^{*}\right), B$ is the right inverse of $H^{\prime}(\hat{z})$. Then the image of $I_{Z}-B \circ H^{\prime}(\hat{z})$ is $\operatorname{Ker} H^{\prime}(\hat{z})$ and hence $\left(R_{4}^{*}\right)$ yields the closedness of the image of $E^{\prime}(\hat{z})$.

The criticality condition $\left(C_{2}\right)$ follows from $\left(C_{2}^{*}\right)$ and Theorem 2.5 .

Conditions $\left(R_{4}\right)$ and $\left(R_{5}\right)$ are immediate. Hence, Theorem 1.1 applied to $(\mathcal{P})^{*}$ yields the existence of nontrivial multipliers $\lambda_{i} \geq 0(i=0, \ldots, p), w^{*} \in\left(L_{m}^{\infty}\right)^{*}$, $v^{*} \in\left(L_{n}^{\infty}\right)^{*}$, and $y^{*} \in Y^{*}$ such that the first equation of (3.2) holds and

$$
\begin{gathered}
w^{*} \in N\left(G(\hat{z}) \mid \sigma_{\infty}(Q)\right) \\
\sum_{i=0}^{p} \lambda_{i} F_{i}^{o}(\hat{z} ; z)+\left\langle y^{*}, K^{\prime}(\hat{z}) z\right\rangle+\left\langle w^{*}, G^{\prime}(\hat{z}) z\right\rangle+\left\langle v^{*}, H^{\prime}(\hat{z}) z\right\rangle \geq 0 \quad \text { for } z \in Z
\end{gathered}
$$

and

$$
\begin{aligned}
& \sum_{i=0}^{p} \lambda_{i} F_{i}^{\prime \prime}(\hat{z} ; y)+\left\langle y^{*}, K^{\prime \prime}(\hat{z})(y)\right\rangle \\
& \quad+\left\langle w^{*}, G^{\prime \prime}(\hat{z})(y)\right\rangle+\left\langle v^{*}, H^{\prime \prime}(\hat{z})(y)\right\rangle \geq 2 \delta^{*}\left(w^{*} \mid V\left(G(\hat{z}), G^{\prime}(\hat{z}) y \mid \sigma_{\infty}(Q)\right)\right)
\end{aligned}
$$

First we shall show that $w^{*}$ and $v^{*}$ are in fact represented in terms of integrable functions.

$$
\begin{aligned}
& \text { Let }(v, w) \in L_{n}^{\infty} \times L_{m}^{\infty} . \text { Set } \\
& \qquad z=z(v, w):=A\left(w-G^{\prime}(\hat{z}) B v\right)+B v,
\end{aligned}
$$


where $A$ is given in $\left(R_{2}^{*}\right)$, which implies

$$
G^{\prime}(\hat{z})\left(A\left(w-G^{\prime}(\hat{z}) B v\right)\right)-\left(w-G^{\prime}(\hat{z}) B v\right) \in T\left(G(\hat{z}) \mid \sigma_{\infty}(Q)\right) .
$$

Hence, by (3.6),

$$
\left\langle w^{*}, G^{\prime}(\hat{z})\left(A\left(w-G^{\prime}(\hat{z}) B v\right)\right)-\left(w-G^{\prime}(\hat{z}) B v\right)\right\rangle \leq 0 .
$$

Due to this inequality and $\left(R_{2}^{*}\right)$, we obtain

$$
\begin{aligned}
&\left\langle w^{*}, G^{\prime}(\hat{z}) z\right\rangle+\left\langle v^{*}, H^{\prime}(\hat{z}) z\right\rangle \\
&=\left\langle w^{*}, G^{\prime}(\hat{z})\left(A\left(w-G^{\prime}(\hat{z}) B v\right)\right)+\left(G^{\prime}(\hat{z}) B v-w\right)+w\right\rangle \\
&+\left\langle v^{*}, H^{\prime}(\hat{z})\left(A\left(w-G^{\prime}(\hat{z}) B v\right)\right)+H^{\prime}(\hat{z})(B v)\right\rangle \\
& \leq\left\langle w^{*}, w\right\rangle+\left\langle v^{*}, v\right\rangle .
\end{aligned}
$$

Substituting $z=z(w, 0)$ and $z=z(0, v)$ into (3.7), respectively, we get that

$$
\sum_{i=0}^{p} \lambda_{i} F_{i}^{o}(\hat{z} ; A(w))+\left\langle y^{*}, K^{\prime}(\hat{z}) A(w)\right\rangle+\left\langle w^{*}, w\right\rangle \geq 0
$$

for $w \in L_{m}^{\infty}$, and

$$
\sum_{i=0}^{p} \lambda_{i} F_{i}^{o}\left(\hat{z} ; A\left(-G^{\prime}(\hat{z}) B v\right)+B v\right)+\left\langle y^{*}, K^{\prime}(\hat{z})\left(A\left(-G^{\prime}(\hat{z}) B v\right)+B v\right)\right\rangle+\left\langle v^{*}, v\right\rangle \geq 0
$$

for $v \in L_{n}^{\infty}$.

Replacing $w$ and $v$ by $(-w)$ and $(-v)$, respectively, in the above inequalities, we also get lower estimates for the linear functionals $w^{*}$ and $v^{*}$. Using the $\Pi$-continuity assumptions of $\left(R_{2}^{*}\right),\left(R_{3}^{*}\right)$, and $\left(R_{4}^{*}\right)$, we obtain that $v^{*}$ and $w^{*}$ are $\Pi$-continuous at the origin. Then, by the Hewitt-Yosida decomposition theorem [27], there exist $\varphi \in L_{m}^{1}$ and $\psi \in L_{n}^{1}$ such that

$$
\left\langle w^{*}, w\right\rangle=\int_{\Omega}\langle\varphi(t), w(t)\rangle d \mu(t) \quad \text { and } \quad\left\langle v^{*}, v\right\rangle=\int_{\Omega}\langle\psi(t), v(t)\rangle d \mu(t)
$$

for all $w \in L_{m}^{\infty}$ and $v \in L_{n}^{\infty}$, respectively. Clearly, these equations reduce (3.7) to (3.4).

Using Corollary 2.7 and (3.11), the second-order necessary condition (3.8) now reduces to (3.5).

Since by (3.11) the functional $w^{*}$ is represented by the $L_{m}^{1}$-function $\varphi$, then $[21$, Theorem 9] and (3.6) yield that the first equation of (3.3) holds true. Furthermore, by replacing $z=y$ in (3.7) and by using the criticality of $y$, we obtain the second equations of (3.2) and (3.3). Therefore, the proof of the theorem is completed. 
Acknowledgment. The authors wish to thank the anonymous referee for the constructive comments and for calling to their attention extra references.

\section{REFERENCES}

[1] V. M. Alekseev, S. V. Fomin, and V. M. Tihomirov, Optimal Control, Nauka, Moscow, 1979 (in Russian).

[2] F. H. Clarke, Optimization and Nonsmooth Analysis, Canadian Math. Soc. Ser. Monogr. Adv. Texts, John Wiley, New York, Chichester, Brisbane, Toronto, Singapore, 1983.

[3] C. Castaing And M. VAladier, Convex-analysis and measurable multifunctions, Lecture Notes in Math. 580, A. Dold and B. Eckmann, eds., Springer-Verlag, Berlin, Heidelberg, New York, 1977.

[4] R. Cominetti, Metric regularity, tangent sets and second-order optimality conditions, Appl. Math. Optim., 21 (1990), pp. 265-287.

[5] A. V. Dmitruk, Quadratic conditions for a Pontryagin minimum in an optimal control problem, linear in the control, with a constraint on the control, Soviet Math. Dokl., 28 (1983), pp. 364-368.

[6] A. V. Dmitruk, Quadratic order conditions of a local minimum for singular extremals in a general optimal control problem, in Differential Geometry and Control, Proc. Sympos. Pure Math. 64, G. Ferreyra et al., eds., AMS, Providence, RI, 1998, pp. 163-198.

[7] A. Ya. Dubovitski and A. A. Milyutin, Extremum problems with constraints, Dokl. Akad. Nauk SSSR, 149 (1963), pp. 759-762 (in Russian). English version available in Soviet Math. Dokl., 4 (1963), pp. 452-455.

[8] A. Ya. Dubovitski And A. A. Milyutin, Extremal problems in the presence of constraints, USSR Comput. Math. and Math. Physics, 5 (1965), pp. 395-453.

[9] F. Hiai AND H. UMegaki, Integrals, conditional expectations, and martingales of multivalued functions, J. Multivariate Anal., 7 (1977), pp. 149-182.

[10] A. D. Ioffe, On some recent developments in the theory of second-order optimality conditions, in Optimization, Lecture Notes in Math. 1405, S. Dolecki, ed., Springer-Verlag, New York, Berlin, 1989, pp. 55-68.

[11] H. KAWASAKI, An envelope-like effect of infinitely many inequality constraints on second-order necessary conditions for minimization problems, Math. Programming, 41 (1988), pp. 7396.

[12] H. KaWASAKI, Second order necessary optimality for minimizing a sup-type function, Math. Programming, 49 (1991), pp, 213-229.

[13] E. S. Levitin, A. A. Milyutin, and N. P. Osmolovskil, Conditions of higher-order for a local minimum in extremal problems with constraints, Russian Math. Surveys, 33 (1978), pp. $97-168$.

[14] Y. Maruyama, Second-order necessary conditions for nonlinear optimization problems in Banach spaces by the use of Neustadt derivative, Math. Japon., 40 (1994), pp. 509-522.

[15] A. A. Milyutin and N. P. Osmolovskis, Calculus of Variations and Optimal Control, AMS, Providence, RI, 1998.

[16] N. P. OsmolovskiI, Necessary and sufficient conditions of a high order for a Pontryagin and a bounded-strong minima in an optimal control problem, Soviet Phys. Dokl., 33 (1988), pp. 883-885.

[17] N. P. Osmolovski, Quadratic conditions for nonsingular extremals in optimal control (a theory), Russian J. Math. Phys., 2 (1994), pp. 487-516.

[18] Zs. PÁles AND V. M. ZeIdAn, Nonsmooth optimum problems with constraints, SIAM J. Control Optim., 32 (1994), pp. 1476-1502.

[19] Zs. PÁles and V. M. Zeidan, Characterization of closed $C$-convex sets in $C\left(T, \mathbb{R}^{n}\right)$, Acta Sci. Math. (Szeged), 65 (1999), pp. 339-357.

[20] Zs. PÁles And V. M. Zeidan, On $L^{1}$-closed decomposable sets in $L^{\infty}$, in Systems Modelling and Optimization (Detroit, MI, 1997), Chapman \& Hall/CRC Res. Notes Math. 396, Boca Raton, FL, 1999, pp. 198-206,

[21] Zs. PÁles AND V. M. ZeIDAN, Characterization of $L^{1}$-closed decomposable sets in $L^{\infty}$, J. Math. Anal. Appl., 238 (1999), pp. 491-515.

[22] Zs. PÁles AND V. ZeIDAN, Optimum problems with certain lower semicontinuous set-valued constraints, SIAM J. Control Optim., 8 (1998), pp. 707-727.

[23] J.-P. Penot, Optimality conditions in mathematical programming and composite optimization, Math. Programming, 67 (1994), pp. 225-245.

[24] R. T. Rockafellar, Convex Analysis, Princeton University Press, Princeton, NJ, 1970. 
[25] R. T. Rockafellar, Integrals which are convex functionals, Pacific J. Math., 24 (1968), pp. 525-539.

[26] R. T. Rockafellar, Integrals which are convex functionals, II, Pacific J. Math., 39 (1971), pp. 439-469.

[27] K. Yosida And E. Hewitt, Finitely additive measures, Trans. Amer. Math. Soc., 72 (1952), pp. $46-66$. 